\title{
Impact of Wind-Induced Scatterers Motion on GB-SAR Imaging
}

\author{
Marc Lort ${ }^{\circledR}$, Member, IEEE, Albert Aguasca ${ }^{\circledR}$, Member, IEEE, Carlos López-Martínez, Senior Member, IEEE, \\ and Xavier Fabregas, Member, IEEE
}

\begin{abstract}
Ground-based synthetic aperture radar (GB-SAR) sensors represent a cost-effective solution for change detection and ground displacement assessment of small-scale areas in real-time early warning applications. GB-SAR systems based on stepped linear frequency modulated continuous wave signals have led to several improvements such as a significant reduction of the acquisition time. Nevertheless, the acquisition time is still long enough to force a degradation of the quality of the reconstructed images because of possible short-term variable reflectivity of the scenario. This reduction of the quality may degrade the differential interferometric detection process. In scenarios where interesting targets are surrounded by vegetation, this is normally related to atmospheric conditions, in particular, the wind. The present paper characterizes the effect of the short-term variable reflectivity in the GB-SAR image reconstruction and evaluates its equivalent blurring effect, the decorrelation introduced in the SAR images, and the degradation of the extracted parameters. In order to validate the results, the study assesses different GB-SAR images obtained with the RISKSAR-X sensor, which has been developed by the Universitat Politècnica de Catalunya.
\end{abstract}

Index Terms-Blurring, coherence, differential synthetic aperture radar (SAR) interferometry (DInSAR), ground-based synthetic aperture radar (GB-SAR), temporal decorrelation.

\section{INTRODUCTION}

D ATA retrieved from orbital synthetic aperture radars (SARs) have been used during the last decades to monitor deformation episodes in large-scale areas of observation by means of differential SAR interferometry [1], [2]. An alternative strategy to monitor small-scale areas is based on ground-based SARs (GB-SAR), which are increasingly being used as early warning systems [3], [4]. The main differences between GBSARs and classical orbital or airborne SARs are the way the aperture is synthesized, the transmitted signal waveform and its power. In GB-SARs, the radar sensor is placed on a mechanical platform of linear displacement, transmitting a triangular

Manuscript received July 21, 2017; revised April 20, 2018 and June 25, 2018; accepted July 29, 2018. This work was supported in part by the Spanish Ministry of Science and Innovation under the projects TIN2014-55413-C2-1-P and TEC2017-85244-C2-2-P, in part by the Unidad de Excelencia Maria de Maeztu MDM-2016-0600, and in part by the research grant BES-2012-060089 (Corresponding author: Marc Lort.)

The authors are with the Remote Sensing Laboratory, Department of Signal Theory and Communications, Universitat Politècnica de Catalunya, Barcelona 08034, Spain (e-mail: marc.lort@tsc.upc.edu; aguasca@tsc.upc.edu; carlos. lopez@tsc.upc.edu; fabregas@tsc.upc.edu).

Color versions of one or more of the figures in this paper are available online at http://ieeexplore.ieee.org.

Digital Object Identifier 10.1109/JSTARS.2018.2863369
FMCW signal of few watts of the output power. Despite its flexibility in terms of rapid deployment and easy operability, some limitations have to be considered, as the reduced length of the aperture that could degrade the cross-range resolution or the long scanning time. High rate stepped linear frequency modulated continuous wave (SLFM-CW) GB-SAR sensors are capable to perform faster scans, reducing the scanning time in the order of seconds. This reduction has led to important improvements in GB-SAR performances for the monitoring of surface deformations by means of coherence maps analysis and persistent scatterer interferometry. An example of such systems is the experimental polarimetric RISKSAR-X sensor developed by the Remote Sensing Laboratory, Universitat Politècnica de Catalunya (UPC) [5]-[7]. Even though the scanning time has been reduced, it is still long enough to take into account the reflectivity changes in the scenario during the scan time. Some alternatives to GB-SAR systems based on mechanical movement can be found in the literature, such as the new generation of GB-SARs based on MIMO technology [8]-[10], which are a promising solution that, by increasing the technological complexity of their architecture, can drastically reduce the scan time.

As it has been pointed out in different experiments and studies [11]-[13], the changes in the scenario might be related to the wind, which may modify its reflectivity in short periods of time if the observed area contains or is surrounded by vegetation [14]. As a result, the quality of the focused images and the extracted parameters is degraded. Some examples of studies performed with GB-SARs in this kind of scenarios can be found in the literature [15]-[19].

The purpose of the present paper is to identify and to understand the phenomena that may reduce the GB-SAR system performances by evaluating the retrieved data, such as coherence and interferometric phase maps. Besides, it is intended to provide a mathematical description of the image blurring by means of the study of the cross-range focusing. The present study takes into account the limited aperture length and the time to perform an acquisition by the GB-SAR. Some of the conclusions presented could be directly applicable to other types of SAR systems, either airborne or orbital.

This paper is organized as follows. Section II presents the main characteristics of the GB-SAR system used in the measurement campaign. Section III is intended to provide a mathematical description of the cross-range focusing process in the GB-SAR and also to justify the effects observed in the retrieved images. In Section IV, a general description of the test site and 
TABLE I

RISKSAR-X SETTING PARAMETERS

\begin{tabular}{lc}
\hline \hline \multicolumn{1}{c}{ System Parameters } & X-Band \\
\hline Carrier Frequency $\left(\mathrm{f}_{0}\right)$ & $9.65 \mathrm{GHz}$ \\
Chirp Sampling Frequency $\left(\mathrm{f}_{\mathrm{s}}\right)$ & $50 \mathrm{MHz}$ \\
Chirp Repetition Frequency & $3.05 \mathrm{KHz}$ \\
Chirp Bandwidth & $100 \mathrm{MHz}$ \\
Deramped Signal Bandwidth & $21 \mathrm{MHz}$ \\
Transmitted Power & $30 \mathrm{dBm}$ \\
3dB Antenna Beamwidth & $27^{\circ}$ \\
Synthetic Aperture Length & $2 \mathrm{~m}$ \\
\hline \hline
\end{tabular}

the dataset employed in this study is given. In Section V, the experimental results are presented. The main conclusions and major remarks of the work are exposed in Section VI.

\section{RISKSAR-X SENSOR}

The instrument employed for the measurements is the RISKSAR-X GB-SAR sensor. It has been used in different applications, such as the monitoring of rock falls in the Montserrat mountain (North-Eastern Spain), the characterization of urban subsidence in Sallent (North-Eastern Spain), or the monitoring of landslides in Canillo (Andorra) [20], [21]. Its characteristics are summarized in Table I and the block diagram of the system architecture is an evolved version of the one presented in [5]. The system operates with a triangular SLFM-CW ramp signal generated by a direct digital synthesizer that is up-converted to the desired frequency by an active frequency multiplier. The transmitted power is delivered by a solid state power amplifier. The receiver unit consists of two parallel low-noise chains with a direct zero-IF demodulator, where a sample of the transmitted signal is used as a local oscillator.

The sensor is fixed on a linear motion unit and operated in a StripMap mode. The scanning technique can be of two types: stop\&go (S\&G) and on-the-fly $(\mathrm{OtF})$. The $\mathrm{S} \& \mathrm{G}$ operation mode divides the aperture length in different fixed, equally spaced positions where the sensor stops and takes an averaged measurement. This technique is the one used by the first GB-SAR systems and implies the need of several minutes to synthesize a 2 m length aperture. In the OtF operation mode, the sensor is measuring continuously, while the linear unit performs the aperture in a few seconds. This operation mode is analogous to the one used in modern GB-SARs [22]. In both cases, it is important to distinguish between the scanning time and the observation time. The observation time is the effective time in which the radar is illuminating the scenario and is related to the total amount of energy transmitted, while the scanning time is the time that the system takes to perform the total aperture. The OtF operation mode has the same observation time and scanning time, but the $S \& G$ mode does not. In our particular case, the $\mathrm{S} \& \mathrm{G}$ mode has the same observation time as that of the OtF mode, i.e., same energy transmitted, but the scanning time is higher due to the computation of the averaging process and the mechanical stop-go-stop movement of the platform. This is of great importance because both modes of operation theoretically have the same signal-to-noise ratio (SNR), but the S\&G mode

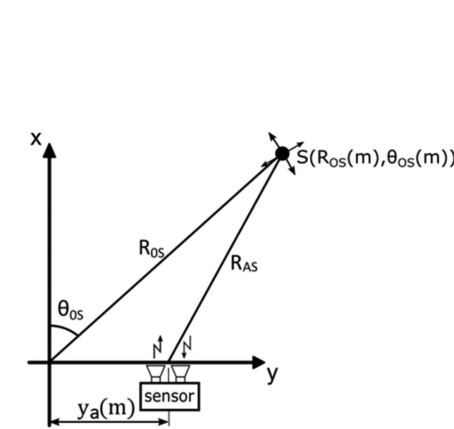

(a)

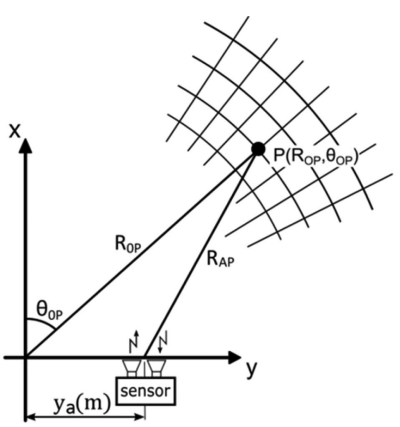

(b)
Fig. 1. Geometry in polar coordinates where the GB-SAR position is $y_{a}(m)$. (a) Scatterer is located at $\left(R_{\mathrm{OS}}(m), \theta_{\mathrm{OS}}(m)\right)$. (b) Pixel associated with the scatterer is $\left(R_{\mathrm{OP}}, \theta_{\mathrm{OP}}\right)$.

is much more affected by the short-term variable reflectivity introduced by moving scatterers, such as the movement of the vegetation, as it is demonstrated in Section V.

The SAR focusing technique is based on the back-projection algorithm (BPA) [23]. The received base-band signal, after the deramping process, is a range compressed by a simple fast Fourier transform. The azimuth focusing by the BPA algorithm, despite its high computational cost, offers a high degree of flexibility with extended images from arbitrary synthetic aperture lengths. The range resolution of the RISKSAR-X is approximately $1.5 \mathrm{~m}$ and the cross-range resolution ranges from $0.75 \mathrm{~m}$ at near range up to approximately $10 \mathrm{~m}$ at the far range.

\section{Mathematical Description of the Cross-RANGe BLURRING EFFECT AND THE REDUCTION OF COHERENCE}

To understand and to quantify the effect in the image reconstruction process of having vegetation with short-term variable reflectivity surrounding the area of interest, the mathematics of the cross-range focusing process has been reformulated. The simplified expression of the transmitted signal in a SAR system working with FM-CW chirp signals is

$$
s_{T}(t)=a(t) \cdot e^{j\left(\omega_{0} t+\frac{1}{2} \alpha t^{2}\right)}
$$

where $\omega_{0}=2 \pi f_{0}, f_{0}$ being the carrier frequency; $\alpha$ is the signal chirp rate; and $t$ is the rapid time related to the propagation of the signal. The rectangular function $a(t)$ can be expressed as

$$
a(t)=\prod\left(\frac{t-\frac{T_{p}}{2}}{T_{p}}\right)
$$

where $a(t)=1$ for $0<t<T_{p}$ and zero otherwise, and $T_{p}$ is the chirp duration. The complex reflectivity function $\Gamma$ describing the scenario depicted in Fig. 1, where it is considered a single point scatterer with variable reflectivity between different transmitted chirps, can be written as

$$
\Gamma(t, m)=\sigma(m) e^{j \phi(m)} \delta\left(t-\tau_{0}(m)\right) .
$$

In OtF mode operation, $m$ coincides with the transmitted chirp period number. The complex reflectivity of the scatterer is $\sigma(m) e^{j \phi(m)}$, and $\tau_{0}(m)$ is the round trip delay from every 
antenna position $y_{a}(m)$ along the aperture to the scatterer. The antenna position is defined as $y_{a}(m)=v \cdot u=v \cdot T_{\mathrm{PRF}} \cdot m$, where $v$ is the speed of the linear unit and the slow time $u=$ $T_{\mathrm{PRF}} \cdot m$. In case of $\mathrm{S} \& \mathrm{G}$ mode operation, $T_{\mathrm{PRF}}$ and $v$ have to be readjusted to an equivalent longer $T_{\mathrm{PRF} \text { _eq }}$ and slower $v_{\text {eq }}$, which takes into account the temporal averaging and the slower motion velocity. The normalized received signal from the illuminated scenario can be expressed as the convolution between (1) and (3), $s_{T} * \Gamma(t, m)$ as follows:

$$
\begin{aligned}
s_{R}(t, m)= & \sigma(m) e^{j \phi(m)} a\left(t-\tau_{0}(m)\right) \\
& \cdot e^{j\left(\omega_{o}\left(t-\tau_{0}(m)\right)+\frac{\alpha}{2} \cdot\left(t-\tau_{0}(m)\right)^{2}\right)} .
\end{aligned}
$$

The transmitter and receiver move along the $y$-axis, as shown in Fig. 1. The round trip delay $\tau_{0}(m)$ can be expressed as

$$
\begin{aligned}
\tau_{0}(m)= & \frac{2 \cdot R_{\mathrm{OS}}(m)}{c} \\
& \times \sqrt{1+\frac{y_{a}(m)^{2}-2 R_{\mathrm{OS}}(m) \cdot y_{a}(m) \cdot \sin \theta_{\mathrm{OS}}(m)}{R_{\mathrm{OS}}^{2}(m)}} \\
& \approx \frac{2}{c}\left(R_{\mathrm{OS}}(m)+\frac{y_{a}(m)^{2}}{2 R_{\mathrm{OS}}(m)}-y_{a}(m) \sin \theta_{\mathrm{OS}}(m)\right)
\end{aligned}
$$

where the scatterer position $\left(R_{\mathrm{OS}}(m), \theta_{\mathrm{OS}}(m)\right)$ can be defined as

$$
\begin{aligned}
& R_{\mathrm{OS}}(m)=\hat{R}_{\mathrm{OS}}+\Delta R_{\mathrm{OS}}(m) \\
& \theta_{\mathrm{OS}}(m)=\hat{\theta}_{\mathrm{OS}}+\Delta \theta_{\mathrm{OS}}(m)
\end{aligned}
$$

where $\left(\hat{R}_{\mathrm{OS}}, \hat{\theta}_{\mathrm{OS}}\right)$ is the nominal position of the scatterer and $\Delta R_{\mathrm{OS}}(m)$ and $\Delta \theta_{\mathrm{OS}}(m)$ are the variation in the position of the scatterer during the slow time $u$.

In a dechirp-on-receive system, the received and transmitted signals are directly mixed [24]. The mixing process generates a zero IF frequency signal that is low-pass filtered

$$
\begin{aligned}
s_{\mathrm{IF}}(t, m)= & \frac{1}{2} \sigma(m) e^{j \phi(m)} a\left(t-\tau_{0}(m)\right) \\
& \cdot e^{j\left(-\alpha \cdot t \cdot \tau_{0}(m)-\omega_{0} \cdot \tau_{0}(m)+\frac{\alpha}{2} \cdot \tau_{0}{ }^{2}(m)\right)} .
\end{aligned}
$$

Notice that the dependence with the transmitted power, antenna gain, receiver power gain, and propagation losses is not included for simplicity. The range compressed version of the received signal is the Fourier transform of $s_{\mathrm{IF}}$ in rapid time

$$
\begin{aligned}
S_{\mathrm{IF}}(\omega, m)= & \mathcal{F} \\
= & \frac{1}{4} \sigma(m) e^{j \phi(m)} \cdot e^{j\left(-\omega_{0} \cdot \tau_{0}(m)+\frac{\alpha}{2} \cdot \tau_{0}{ }^{2}(m)\right)} \\
& \cdot e^{-j\left(\omega+\alpha \tau_{0}(m)\right) \cdot \tau_{0}(m)} \cdot A\left(\omega+\alpha \tau_{0}(m)\right)
\end{aligned}
$$

where $A(\omega)$ is the Fourier transform of $a(t)$

$$
A(\omega)=T_{P} \sin c\left(\frac{T_{p}}{2} \omega\right) e^{-j \omega \frac{T_{p}}{2}} .
$$

If it is assumed that the energy is mainly around the maximum in the point spread function, which can be expressed as $\omega=-\alpha \tau_{0}(m)$, the term $e^{-j\left(\omega+\alpha \tau_{0}(m)\right) \cdot \tau_{0}(m)}$ in (8) can be neglected. In our particular case, the chirp duration is $0.16 \mathrm{~ms}$, so it can be assumed that the range compressed signal is not affected by moderate disturbances and the impulse response is preserved (static target approximation). After the range compression, the cross-range image reconstruction is performed by means of the BPA. Any pixel $p(R, \theta)$ of the image is reconstructed as follows:

$$
p(R, \theta)=\sum_{m=0}^{M-1} S_{\mathrm{IF}}(\omega, m) \cdot e^{j\left(\omega_{0} \tau(m)-\frac{\alpha}{2} \cdot \tau^{2}(m)\right)}
$$

where $\tau(m)$ is the round trip delay, $\omega$ is a function of $R$ by means of $\omega=\alpha \cdot \tau(m)$, and $M$ is the total number of antenna positions or transmitted chirp periods along the aperture. Indeed, (10) corresponds to the matched filter for a given pixel at $(R, \theta)$, i.e., the convolution of the received signal with the complex conjugated of the expected response for a given pixel.

The expression of the $p(R, \theta)$ function can be employed to find the equivalent cross-range response of the GB-SAR. Assuming that there is only one scatterer located at $\left(R_{\mathrm{OS}}, \theta_{\mathrm{OS}}\right)$, the reconstructed pixel $p\left(R_{\mathrm{OP}}, \theta\right)$ along the $\theta$-axis can be evaluated

$$
p\left(R_{\mathrm{OP}}, \theta\right) \cong K \cdot \sum_{m=0}^{M-1} \sigma(m) e^{j \phi(m)} \cdot e^{j \omega_{0} \cdot\left(\tau_{0}(m)-\tau_{p}(m)\right)}
$$

where $\tau_{p}(m)$ is the round trip delay from every antenna position $y_{a}(m)$ to the pixel at $(R, \theta)$ and its expression is geometrically similar to $\tau_{0}(m)$. The terms $e^{j \frac{\alpha}{2} \tau_{0}{ }^{2}(m)}$ and $e^{-j \frac{\alpha}{2} \tau_{p}{ }^{2}(m)}$, which are related to the residual video phase error, have not been included because their contribution to the reconstruction of $p$ is negligible. It has been assumed that $K=\frac{1}{4} A\left(\omega+\alpha \tau_{0}(m)\right)$ is constant during the evaluated scanning time. This is equivalent to assume no range cell migration in the cross-range dimension, which is a feasible approximation if the total aperture length is a fraction of the scatterer range.

The reconstructed pixel in (11) can be expressed as

$$
\begin{aligned}
p\left(R_{\mathrm{OP}}, \theta\right) \cong & \sum_{m=0}^{M-1} K \sigma(m) e^{j \phi(m)} \\
& \cdot e^{j \frac{4 \pi}{\lambda} \cdot\left(R_{\mathrm{OS}}(m)-R_{\mathrm{OP}}\right)} \\
& \cdot e^{j \frac{4 \pi \cdot y_{a}^{2}(m)}{\lambda} \cdot\left(\frac{1}{R_{\mathrm{OS}}(m)}-\frac{1}{R_{\mathrm{OP}}}\right)} \\
& \cdot e^{j \frac{4 \pi \cdot y_{a}(m)}{\lambda} \cdot\left(\sin \theta-\sin \theta_{\mathrm{OS}}(m)\right)} .
\end{aligned}
$$

Assuming that the pixels under study are at the same range as the scatterer $R_{\mathrm{OP}}=\hat{R}_{\mathrm{OS}}$, the expression of (12) can be rewritten as

$$
\begin{aligned}
& p\left(\hat{R}_{\mathrm{OS}}, \theta\right) \cong \sum_{m=0}^{M-1} K \sigma(m) e^{j \phi(m)} \\
& \cdot e^{j \frac{4 \pi}{\lambda} \cdot \Delta R_{\mathrm{OS}}(m)} \cdot e^{j \frac{4 \pi \cdot y_{a}^{2}(m)}{\lambda} \cdot\left(\frac{1}{\hat{R}_{\mathrm{OS}}+\Delta R_{\mathrm{OS}}(m)}-\frac{1}{R_{\mathrm{OP}}}\right)} \\
& \cdot e^{j \frac{4 \pi \cdot y_{a}(m)}{\lambda} \cdot\left(\sin \theta-\sin \theta_{\mathrm{OS}}(m)\right)}
\end{aligned}
$$


where the term $\frac{1}{\hat{R}_{\mathrm{OS}}+\Delta R_{\mathrm{OS}}(m)}$ can be expressed as

$$
\begin{aligned}
\frac{1}{\hat{R}_{\mathrm{OS}}+\Delta R_{\mathrm{OS}}(m)} & =\frac{1}{\hat{R}_{\mathrm{OS}}}\left(\frac{1}{1+\frac{\Delta R_{\mathrm{OS}}(m)}{\hat{R}_{\mathrm{OS}}}}\right) \\
& \cong \frac{1}{\hat{R}_{\mathrm{OS}}} \cdot\left(1-\frac{\Delta R_{\mathrm{OS}}(m)}{\hat{R}_{\mathrm{OS}}}\right) \\
& =\frac{1}{\hat{R}_{\mathrm{OS}}}-\frac{\Delta R_{\mathrm{OS}}(m)}{\hat{R}_{\mathrm{OS}}^{2}} .
\end{aligned}
$$

Substituting (14) into (13) and assuming that $\hat{R}_{\mathrm{OS}}^{2} \gg$ $\Delta R_{\mathrm{OS}}(m)$ yield the simplified expression

$$
\begin{aligned}
p\left(\hat{R}_{\mathrm{OS}}, \theta\right) \cong & \sum_{m=0}^{M-1} K \sigma(m) e^{j \phi(m)} \\
& \cdot e^{j \frac{4 \pi}{\lambda} \cdot \Delta R_{\mathrm{OS}}(m)} \\
& \cdot e^{j \frac{4 \pi \cdot y a(m)}{\lambda} \cdot\left(\sin \theta-\sin \theta_{\mathrm{OS}}(m)\right)} .
\end{aligned}
$$

Taking into account that for long $\hat{R}_{\mathrm{OS}}$ the equivalent angle variation of the scatterer position $\Delta \theta_{\mathrm{OS}}(m)$ is negligible, the expression of (15) can be considered as follows:

$$
\begin{aligned}
p\left(\hat{R}_{\mathrm{OS}}, \theta\right) \cong & \sum_{m=0}^{M-1} K \sigma(m) e^{j \phi(m)} \\
& \cdot e^{j \frac{4 \pi}{\lambda} \cdot \Delta R_{\mathrm{OS}}(m)} \\
& \cdot e^{j \frac{4 \pi \cdot y a(m)}{\lambda} \cdot\left(\sin \theta-\sin \hat{\theta}_{\mathrm{OS}}\right) .}
\end{aligned}
$$

Defining $G(m)=K \sigma(m) e^{j \phi(m)} \cdot e^{j \frac{4 \pi}{\lambda} \cdot \Delta R_{\mathrm{OS}}(m)}$ and considering the total length of the aperture $L=M \cdot T_{\mathrm{PRF}} \cdot v,(16)$ can be rewritten as

$$
p\left(\hat{R}_{\mathrm{OS}}, \theta\right) \cong \sum_{m=0}^{M-1} G(m) \cdot e^{j \frac{4 \pi \cdot m \cdot L}{\lambda \cdot M} \cdot\left(\sin \theta-\sin \hat{\theta}_{\mathrm{OS}}\right)} .
$$

Assuming a new polar coordinate system, the following two variables can be defined:

$$
\begin{gathered}
\beta_{\mathrm{OS}} \cong \frac{2 L}{\lambda} \sin \hat{\theta}_{\mathrm{OS}} \\
\beta=\frac{2 L}{\lambda} \sin \theta
\end{gathered}
$$

where the $\beta$ coordinate is a sinusoidal function of the polar angle $\theta$. Applying the new polar coordinate system, the reconstructed pixel is

$$
p\left(\hat{R}_{\mathrm{OS}}, \beta\right) \cong \sum_{m=0}^{M-1} G(m) \cdot e^{j \frac{2 \pi \cdot m}{M} \cdot\left(\beta-\beta_{\mathrm{OS}}\right)}
$$

where the pixel reconstruction along the equivalent $\beta$-axis in (19) has an equivalent expression of a discrete Fourier transform (DFT) of the sequence $G(m)$. In case of having multiple scatterers at the same range $R$ and different $\beta_{\mathrm{OS} \_n}$, the expression of (19) can be rewritten as

$$
p\left(\hat{R}_{\mathrm{OS}}, \beta\right) \cong \sum_{m=0}^{M-1} \sum_{n=0}^{N-1} G_{n}(m) \cdot e^{j \frac{2 \pi \cdot m}{M} \cdot\left(\beta-\beta_{\mathrm{OS} \_n}\right)}
$$
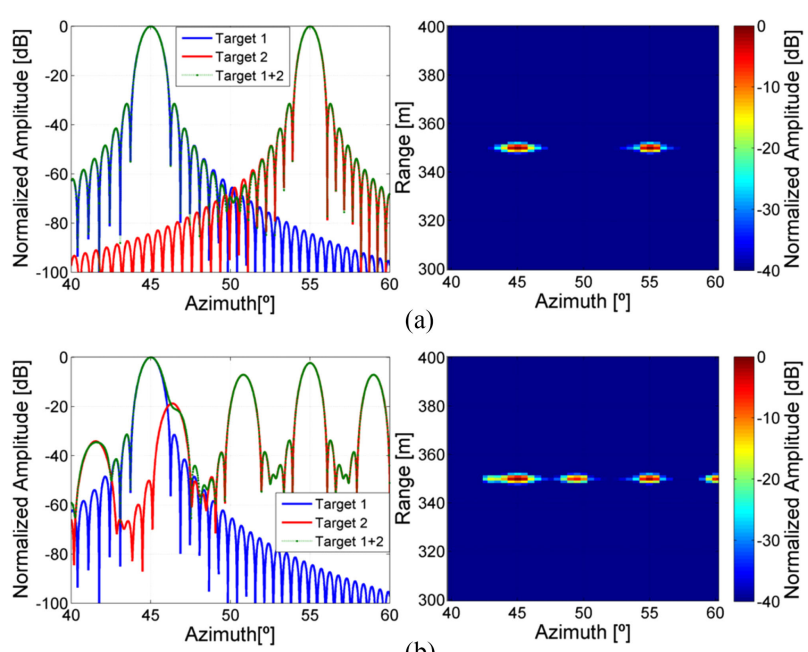

(b)
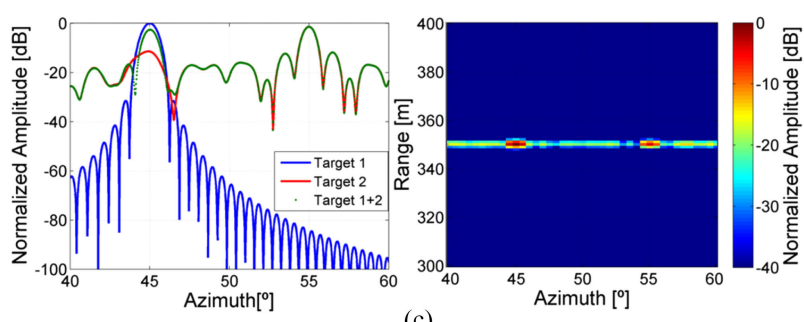

(c)
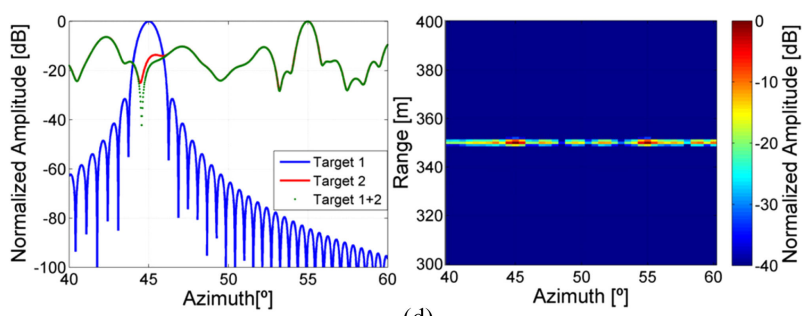

(d)

Fig. 2. Two simulated targets. Left: cut of the targets in polar coordinates. Right: SLC images obtained with BPA in polar coordinates. Scatterer 1 is a static target and scatterer 2 is a (a) static target. (b) Target with a sinusoidal motion in the range where the phase $\phi(m)$ of its reflectivity has a period of $15 \pi$ radians during the slow time $u$. (c) Target with a random motion where the amplitude $\sigma(m)$ of its reflectivity has been modeled as a real random variable that is normally distributed with $\mu=1$ and $\sigma_{\varnothing}^{2}(m)=5$, and the phase $\phi(m)$ as a real random variable that is normally distributed with $\mu=1$ and $\sigma_{\varnothing}^{2}(m)=1$. (d) Distributed target inside the resolution cell modeled as a stochastic process with the same parameters as (c).

where $N$ is the total number of scatterers at different angles (different $\beta_{\mathrm{OS} \_n}$ ). In case of having a stable scatterer, $G(m)=$ constant, the cross-range response along the $\beta$-axis is a sinc function and its extension depends on the ratio $\lambda / L$. As expected, the cross-range resolution of a GB-SAR depends on the length of the aperture. On the other hand, a moving scatterer means that $G(m) \neq$ constant and, as it will be demonstrated mathematically and experimentally in Sections III and V, respectively, it implies image blurring and decorrelation between images due to the movement of the vegetation.

\section{A. Image Blurring Characterization}

Starting from the mathematical description developed previously, the present section studies the blurring effect in the retrieved SAR images. The simulations presented in Fig. 2 are 
intended to provide a graphic illustration of the effect that produces in the single look complex (SLC) images the fact of having moving scatterers with variable complex reflectivity during the acquisition time. As will be shown, the neighboring cells of a moving scatterer may be altered depending on its motion, which could be analogous to have vegetation around the area of interest altering the pixels under study. Taking advantage of the similarity of the pixel reconstruction to a DFT, the qualitative effect of a moving scatterer in slow time can be studied by means of the mathematical description presented in this section. Fig. 2(a) shows the simulation along the $\beta$-axis of (20), where two static scatterers are located at $\hat{\theta}_{\mathrm{OS}_{-} 1}=45^{\circ}$ and $\hat{\theta}_{\mathrm{OS}-2}=55^{\circ}$. As expected, two sinc functions are obtained. Thus, for this particular case, the reconstructed pixel in (20) can be expressed as

$$
\begin{aligned}
p\left(\hat{R}_{\mathrm{OS}}, \beta\right) \cong & \sum_{m=0}^{M-1} G_{\mathrm{perm} 1}(m) \cdot e^{j \frac{2 \pi \cdot m}{M} \cdot\left(\beta-\beta_{\left.\mathrm{OS} \_ \text {perm } 1\right)}\right)} \\
& +\sum_{m=0}^{M-1} G_{\mathrm{perm} 2}(m) \cdot e^{j \frac{2 \pi \cdot m}{M} \cdot\left(\beta-\beta_{\mathrm{OS} \_ \text {perm } 2}\right)} \\
= & \operatorname{sinc}\left(\beta-\beta_{\left.\mathrm{OS} \_ \text {perm } 1\right)}\right. \\
& +\sin \mathrm{c}\left(\beta-\beta_{\text {OS_perm } 2)}\right.
\end{aligned}
$$

where $G_{\text {perm } 1}(m)$ and $G_{\text {perm } 2}(m)$ are constant during the aperture, generating the two sinc functions centered at $\beta_{\mathrm{OS} \text { _perm } 1}$ and $\beta_{\mathrm{OS} \text { _perm2 }}$, respectively.

In another way, the case of considering one scatterer that presents a short oscillating periodic movement in the range direction is particularly interesting. The recovered complex reflectivity of the associated pixel, and also the reflectivity of neighboring pixels, will be affected in amplitude and phase. Fig. 2(b) shows the result of two simulated scatterers; scatterer 1 is a static target, while scatterer 2 presents a sinusoidal motion in the range with a period shorter than the total time of the acquisition. The phase $\phi_{\text {periodic }}(m)$ of the complex reflectivity of scatterer 2 has been modeled as a sinusoidal function. It can be observed, as it was pointed out in [25], that this range motion has an equivalent phase modulation in the complex reflectivity that introduces replicas along the $\beta$-axis. This causes the appearance of nonexisting scatterers (ghost scatterers) in the SLC image. Since the reflectivity map is changed due to the apparition of ghost scatterers, which depends on the periodic movement of the scatterer during the acquisition time, the coherence maps may be modified. In practice, this may provoke an alteration in the number of coherent pixels. In this case, (20) can be rewritten as

$$
\begin{aligned}
p\left(\hat{R}_{\mathrm{OS}}, \beta\right) \cong & \sum_{m=0}^{M-1} G_{\text {perm }}(m) \cdot e^{j \frac{2 \pi \cdot m}{M} \cdot\left(\beta-\beta_{\mathrm{OS} \text { _perm }}\right)} \\
& +\sum_{m=0}^{M-1} G_{\text {periodic }}(m) \cdot e^{j \frac{2 \pi \cdot m}{M} \cdot\left(\beta-\beta_{\text {OS_periodic }}\right)} \\
= & \sin \mathrm{c}\left(\beta-\beta_{\text {OS_perm }}\right)+\text { is }\left(\beta-\beta_{\text {OS_periodic }}\right)
\end{aligned}
$$

where the term $G_{\text {perm }}(m)$ is constant during the aperture, while the term $G_{\text {periodic }}(m)=K_{\text {periodic }} \sigma_{\text {periodic }}(m) e^{j \phi_{\text {periodic }}(m)}$. $e^{j \frac{4 \pi}{\lambda} \cdot \Delta R_{0 \mathrm{~S}_{\text {periodic }}}(m)}$ varies with the movement of scatterer 2. Thus, the reconstruction of a pixel associated with a scatterer can be expressed as the sum of the response of the desired scatterer and an interference signal is $\left(\beta-\beta_{\mathrm{OS} \text { _periodic }}\right)$, which is the result of a phase modulation in the frequency domain that introduces spatial replicas in the SLC image. The separation of the replicas depends on the periodic movement of the target during the acquisition time.

In contrast, in case of nonperiodic motion scatterers, the complex reflectivity of moving scatterers in a resolution cell can be modeled as a Brownian motion affecting to its amplitude and phase [26]. Nevertheless, if the scatterer presents a stochastic motion during slow time $u$, the amplitude $\sigma(m)$ and phase $\phi(m)$ of the reflectivity will be modified, not only affecting the resolution cell but also the neighboring cells at the same equivalent range. This means that the fact that neighboring cells are affected may introduce a blurring effect in the cross-range direction. Fig. 2(c) shows two simulated scatterers; scatterer 1 is a static target, while scatterer 2 presents a random motion in slow time, and its complex reflectivity $\sigma_{\text {random }}(m) e^{j \phi_{\text {random }}(m)}$ has been modeled as a stochastic process. The energy tails of scatterer 2 have been increased because of its inherent stochastic motion. This causes the blurring effect in the cross-range of the SLC images due to the dispersion of the energy along the $\beta$-axis. It is equivalent to the effect of defocusing and blurring in SAR images for scenarios with oceanic waves [27]. This effect is only present in the cross-range direction because in a GB-SAR the Doppler bandwidth of the system is on the order of tens of $\mathrm{Hz}$, which is comparable to the frequency spreading of the energy of moving scatterers. On the contrary, the increment in frequency that justifies a change of the cell in the range direction is on the order of tens of $\mathrm{kHz}$, which is too high compared to the frequency spreading of the energy of moving scatterers. Besides, as the observed reflectivity is the sum of the contributions of all the scatterers (in this case scatterers 1 and 2), the energy dispersion implies that adjacent cells in the same range, although being associated with static scatterers, will show a variable reflectivity between consecutive images. This provokes time decorrelation and, in consequence, a reduction of the coherence between the cells. It can be appreciated in the simulation in Fig. 2(c), where a reduction in the main lobe of the static scatterer is observed by comparing the blue and the green lines. Consequently, a decorrelation between images arises and degradation in the coherence maps between consecutive images can be observed.

For the case of having a permanent scatterer and a scatterer that moves randomly, (20) can be expressed as

$$
\begin{aligned}
p\left(\hat{R}_{\mathrm{OS}}, \beta\right) \cong & \left.\sum_{m=0}^{M-1} G_{\mathrm{perm}}(m) \cdot e^{j \frac{2 \pi \cdot m}{M} \cdot\left(\beta-\beta_{\mathrm{OS}} \mathrm{perm}\right.}\right) \\
& \left.+\sum_{m=0}^{M-1} G_{\mathrm{random}}(m) \cdot e^{j \frac{2 \pi \cdot m}{M} \cdot\left(\beta-\beta_{\mathrm{OS}} \mathrm{random}\right.}\right) \\
= & \operatorname{sinc}\left(\beta-\beta_{\mathrm{OS}_{\mathrm{perm}}}\right)+\mathrm{is}\left(\beta-\beta_{\mathrm{OS}_{\mathrm{random}}}\right)
\end{aligned}
$$

where $G_{\text {random }}(m)=K_{\text {random }} \quad \sigma_{\text {random }}(m) e^{j \phi_{\text {random }}(m)}$. $e^{j \frac{4 \pi}{\lambda} \cdot \Delta R_{O S \text { random }}(m)}$ varies during the aperture, generating the 
uncorrelated interference signal is $\left(\beta-\beta_{\text {OS_random }}\right)$ along the $\beta$-axis, which is related to the cross-range frequency spreading.

Finally, Fig. 2(d) shows the case in which scatterer 2 is a distributed target, consisting of different elements inside the resolution cell that move randomly during the acquisition time. This is analogous to the example shown in Fig. 2(c) and (23), where the amplitude $\sigma_{\text {random }}(m)$ and phase $\phi_{\text {random }}(m)$ of the reflectivity are modeled stochastically, but in this case, the complex reflectivity is modeled as the sum of the complex reflectivities of the different elements inside the resolution cell

$$
\begin{aligned}
p\left(\hat{R}_{\mathrm{OS}}, \beta\right) \cong & \sum_{m=0}^{M-1} G_{\mathrm{perm}}(m) \cdot e^{j \frac{2 \pi \cdot m}{M} \cdot\left(\beta-\beta_{\mathrm{OSprm}}\right)} \\
& \left.+\sum_{k=0}^{K-1} \sum_{m=0}^{M-1} G_{\text {random }}(m) \cdot e^{j \frac{2 \pi \cdot m}{M} \cdot\left(\beta-\beta_{\mathrm{OS}}\right. \text { random }}\right) \\
= & \operatorname{sinc}\left(\beta-\beta_{\mathrm{OS}_{\mathrm{perm}}}\right)+\sum_{k=0}^{K} \text { is }\left(\beta-\beta_{\mathrm{OS}_{\mathrm{random}}}\right)
\end{aligned}
$$

where $K$ is the number of elements in the resolution cell that conform the distributed scatterer.

\section{B. Image Decorrelation Characterization}

The decorrelation between images, which is introduced by the fact of having a short-term variable reflectivity during the acquisition time, is analyzed in this section by means of the complex coherence. The complex coherence is defined as the correlation coefficient of two SAR images $S_{1}$ and $S_{2}$ [28]

$$
\gamma=\frac{E\left\{S_{1} S_{2}^{*}\right\}}{\sqrt{E\left\{\left|S_{1}\right|^{2}\right\} E\left\{\left|S_{2}\right|^{2}\right\}}}
$$

where $E\{x\}$ is the mathematical expectation of $x$ and $*$ is the complex conjugate. The magnitude of (25) $|\gamma|$ is called coherence, and its argument is the effective phase difference between reflectivity maps. The simplified expression for the differential interferometric coherence has to include a new term $\gamma_{\text {blur }}$ to take into account the blurring effect

$$
\gamma=\gamma_{t} \gamma_{\text {th }} \gamma_{\text {other }} \gamma_{\text {blur }}
$$

The term $\gamma_{\text {other }}$ takes into account other decorrelation factors as exposed in [29] and $\gamma_{t}$ considers the changes in the backscattered signal due to different instants of measurement. The thermal noise contribution $\gamma_{\mathrm{th}}$ depends on the SNR and $\gamma_{\text {blur }}$ accounts for the cross-range image blurring. As $\gamma_{\text {th }}$ is negligible because of the high power transmitted and the reduced dimensions of the illuminated scene [30], the degradation of the coherence $\gamma$ can be related to the temporal decorrelation occurred between different acquisitions and the blurring effect in the cross-range direction during the scanning time. Assuming that the scenario has not changed during the short period of time between measurements, which means that there is no temporal decorrelation between images, the blurring effect $\gamma_{\text {blur }}$ is, in our case, mainly responsible for the decorrelation between images

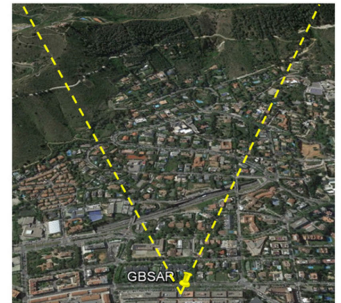

(a)

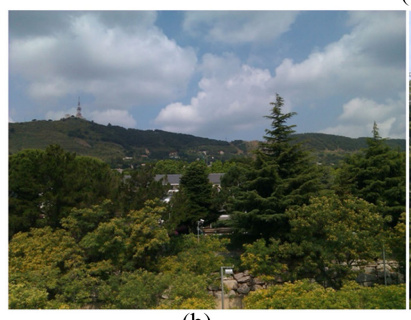

(b)

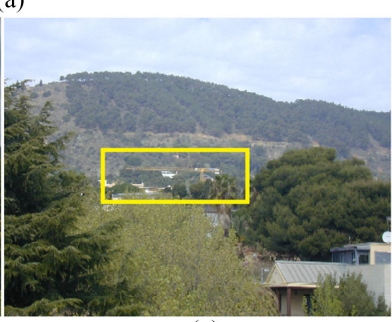

(c)
Fig. 3. Test site. (a) Aerial view of the test site from Google Earth. (b) Photograph of the field of view of the GB-SAR. (c) Photograph of a crane in the test site.

and the largest contributor to the degradation of the coherence. This term, in contrast with $\gamma_{t}$, shows a correlation with the wind conditions. Besides, as shown in Fig. 2 and assessed from (20), the effect of energy spreading along the cross-range axis can be considered as an interference signal, which is assumed totally uncorrelated with respect to the signal from the cell under study. In this case, the correlation between the response of two signals $z_{1}=s$ and $z_{2}=s+$ is, where $s$ is the signal and is the interference signal, can be evaluated as

$$
\begin{aligned}
\gamma_{\text {blur }} & =\frac{E\left\{z_{1} z_{2}^{*}\right\}}{\sqrt{E\left\{\left|z_{1}\right|^{2}\right\} E\left\{\left|z_{2}\right|^{2}\right\}}} \\
& =\frac{E\left\{s(s+\text { is })^{*}\right\}}{\sqrt{E\left\{s^{2}\right\}\left(E\left\{s^{2}\right\}+E\left\{\text { is }^{2}\right\}\right)}}=\frac{1}{\sqrt{1+\frac{1}{\text { SIR }_{e}}}}
\end{aligned}
$$

where $\mathrm{SIR}_{e}=E\left\{s^{2}\right\} / E\left\{\mathrm{is}^{2}\right\}$ is the signal to interference ratio and $s$ is uncorrelated with the interference signal is. It can be seen from (27) that the higher the equivalent interference of the adjacent cells, the lower the $\mathrm{SIR}_{e}$, which implies a reduction of $\gamma_{\text {blur }}$.

To assess the image blurring effect, the study of the evolution of the decorrelation along a temporal sequence of acquisitions constitutes the subject of the measurement campaign presented in Section V. The appearance of ghost scatterers and the crossrange blurring effect introduced by the motion of the scatterers is also analyzed in that section.

\section{TeSt Site DESCRIPTION AND DATASET}

The test site is the Eastern side of the Collserola hill, surrounding the city of Barcelona, Spain $\left(41^{\circ} 230 \mathrm{~N}, 2^{\circ} 060 \mathrm{E}\right)$. It is a heterogeneous scenario including prominent scatterers, low vegetation, and wooded and urban areas, see Fig. 3. The yellow lines define the observable area with an extension of roughly $1.2 \times 1.5 \mathrm{~km}^{2}$. 


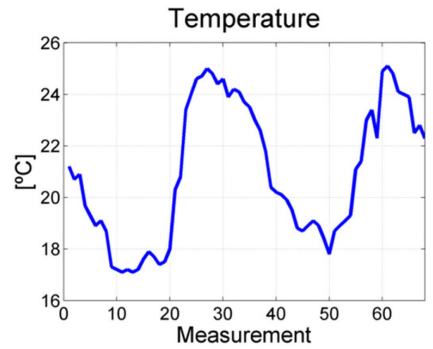

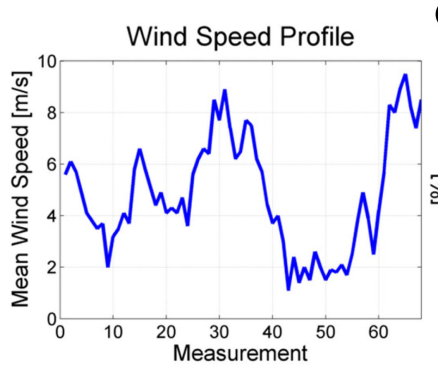

(b) (a)

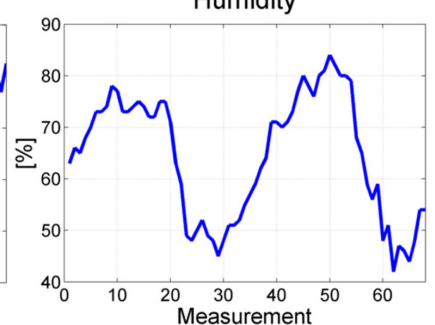

(c)
Fig. 4. Atmospheric parameters information during the measurement campaign (www.meteo.cat). (a) Temperature. (b) Mean wind speed. (c) Humidity.

The UPC GB-SAR sensor was operated in two different modes, $\mathrm{S} \& \mathrm{G}$ and OtF modes, performing measurements of different scanning times. A sequence of 68 measurements was acquired from 18:00 h local time in July 26th to 18:00 h in July 27 th with a time step of $40 \mathrm{~min}$. Each measurement was composed of three different scans with an aperture length of $2 \mathrm{~m}$. The first scan was performed in the OtF mode with a duration of $6 \mathrm{~s}$ and the following two scans were performed in the $\mathrm{S} \& \mathrm{G}$ MID and S\&G SLOW modes with a duration of 60 and $600 \mathrm{~s}$, respectively. It has been ensured that the three different scans have the same total transmitted energy.

During the measurement campaign, the weather conditions were typical of the summer season in the west Mediterranean. The most important atmospheric parameters are presented in Fig. 4: temperature, relative humidity, and mean wind speed. Two periods of strong wind together with high temperatures and low humidity can be noticed.

\section{EXPERIMENTAL RESULTS}

In this section, the most important results of the measurement campaign are presented. They will facilitate the analysis of the blurring effect and the coherence degradation between images pointed out previously. These two main effects, due to moving scatterers in the scenario during the scanning time, have a direct relationship with atmospheric phenomena, concretely the wind.

\section{A. Effect of Oscillating Scatterers in the Retrieved Images}

To evaluate the effect in the retrieved images of an oscillating scatterer in the range direction during the scanning time, the behavior of a crane located in the Collserola test site has been studied, see Fig. 3(c). During the measurements, it evidenced oscillatory movements due to the effect of the wind and normal operation of a flexible structure. The RISKSAR-X sensor was

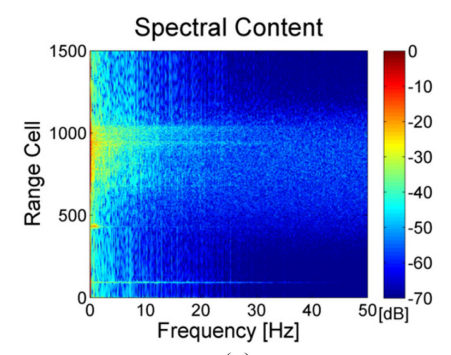

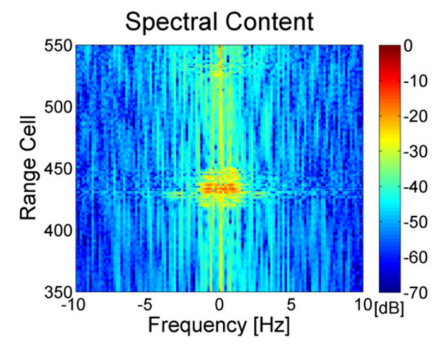

(b)

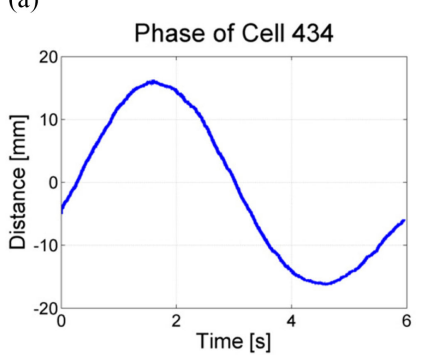

(c)
Fig. 5. (a) Normalized spectrogram of the range cells. (b) Zoom to the range cells of the crane in the normalized spectrogram. (c) Phase of the cell 434 corresponding to the crane during the scanning time.

located in a fixed position to perform an equivalent radar cut. The system scanning time was approximately $6 \mathrm{~s}$. Fig. 5(a) shows the spectrogram of the range cut of a measurement where the range cell 434 corresponds to the crane. During the measurement, there was strong wind, which can be noticed by the energy spreading along the frequency axis in the spectrogram. Fig. 5(b) shows a zoom to the range cells corresponding to the crane in the normalized spectrogram. The crane is also spread along the frequency axis due to its motion during the acquisition time. The phase of the pixel of the crane is related to the distance $R_{\mathrm{AS}}(m)$ from the sensor to the scatterer as follows:

$$
\phi_{\text {crane }}=\frac{4 \pi}{\lambda} \cdot R_{\mathrm{AS}}(m) .
$$

According to (28), the evolution during the scanning time of the phase of the range cell of the crane was extracted and evaluated in Fig. 5(c). The $Y$-axis has been converted to relative distance in millimeters to show the absolute movement of the scatterer. The retrieved phase reveals that the center of the phase of the pixel associated with the crane was oscillating around $30 \mathrm{~mm}$ in the range direction during the scanning time, and hence a sinusoidal curve has been obtained.

Fig. 6 shows the obtained SLC image operating the system in the $\mathrm{S} \& \mathrm{G}$ operation mode. The effect of the movement of the crane in the focused image is clearly seen. Since the scanning time is $60 \mathrm{~s}$, the phase of the pixel corresponding to the crane presents several periods of oscillation during the measurement. This introduces a phase modulation in its complex reflectivity that results in the appearance of replicas along the cross-range direction, as illustrated in the simulations in Fig. 2(b) in Section III. The number of replicas and their angular separation depends on the oscillating period of the scatterer with respect to the acquisition time. 


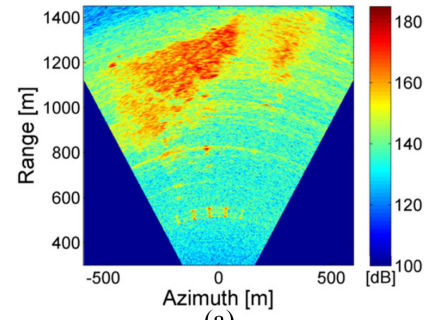

(a)

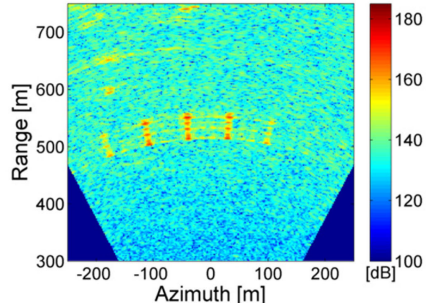

(b)
Fig. 6. (a) SLC image in Cartesian coordinates with replicas along the crossrange of an oscillating crane in the scenario. (b) Zoom of the replicas of the crane in the SLC image.

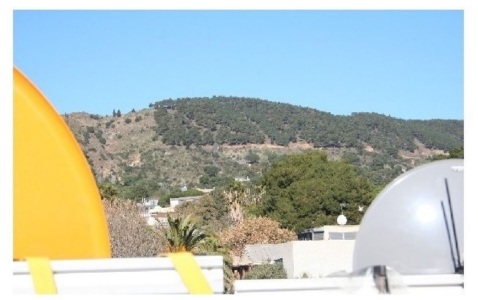

(a)

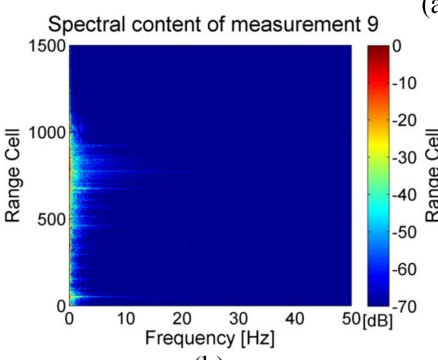

(b)

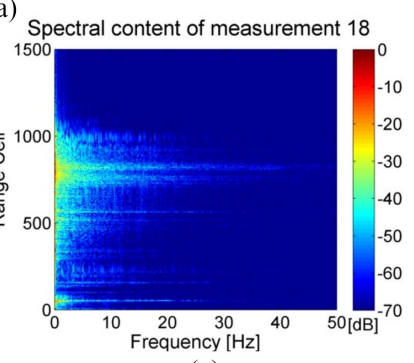

(c)
Fig. 7. (a) Location of two high directive parabolic antennas pointing to the Collserola test site. Normalized spectrogram of the range cells for two different mean wind speeds (b) $v_{\mathrm{wind}}=0.8 \mathrm{~m} / \mathrm{s}$ and (c) $v_{\mathrm{w} \text { ind }}=4.9 \mathrm{~m} / \mathrm{s}$.

\section{B. Effect of Windblown in the Retrieved Images}

In order to evaluate the effect on the quality of the image of the random, short-term variable complex reflectivity $\sigma(m) e^{j \phi(m)}$ of a generic scatterer, a specific experiment was performed. The RISKSAR-X sensor was again used as a one-dimensional radar using a pair of high directive antennas with equivalent radar beamwidth of approximately $2^{\circ}$. The antennas were pointed toward the wooded area, Fig. 7(a). The scans were of short intervals of time of roughly $6 \mathrm{~s}$ and were performed in different moments of the same day with contrasted wind conditions. Fig. 7(b) and (c) shows the spectrogram of the range cut in two times of the day, with different mean wind speeds of 0.8 and $4.9 \mathrm{~m} / \mathrm{s}$, respectively. The normalized spectrogram under higher wind speed presents an important energy dispersion in the frequency domain with respect to the one with lower wind speed, which is consistent with the simulations in Fig. 2(c) in Section III.

The power spectral density (PSD) of the range-compressed signal from two different cells (nos. 351 and 800) is studied under distinct wind conditions. The first cell is identified as a prominent scatterer, while the second corresponds to a vegetated, wooden area. In Fig. 8, the PSD of these cells is compared

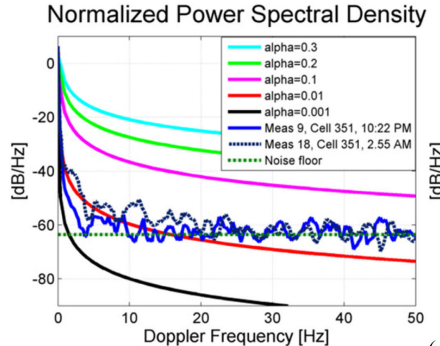

Normalized Power Spectral Density

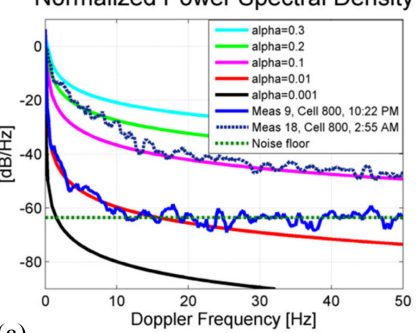

(a)

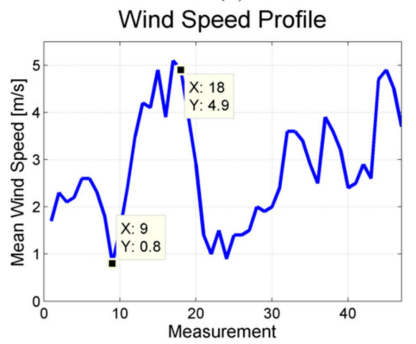

(b)

Fig. 8. Comparison of K-PSD model distribution with PSD of cells no. (a) 351 corresponding to a static scatterer and (b) 800 corresponding to a wooded area for (c) two different instants of the day with different average wind speeds, 0.8 and $4.9 \mathrm{~m} / \mathrm{s}$.

with the model for the Doppler spectrum of a windblown radar ground clutter called K-PSD [14].

The K-PSD model is highly appropriate for characterizing clutter spectra from windblown vegetation. Different PSD shapes are obtained for different values of $\alpha$, which are related to the movement of the vegetation. The parameter $\alpha$, which has been adjusted to match the wind strength, controls the scaling of the exponential slope, the shape of the peak, and the global variance of the clutter PSD.

The PSD of both cells in Fig. 8 is quite different. While cell 351 shows a delta shape, as expected for a prominent static scatterer, cell 800 presents an exponential decay response with notable influence of the measurement instant. Both images, Fig. 8(a) and (b), show the noise floor of the system, and the equivalent frequency bandwidth of the PSD is clearly seen, which is wide enough to ensure a change in the backscatter reflectivity of the cell during the typical scanning time of the GB-SAR.

The energy dispersion of the equivalent reflectivity of these distributed scatterers is wide enough, on the order of tens of $\mathrm{Hz}$, to degrade the focusing process, with an equivalent cross-range blurring effect, as pointed in Section III. The focusing degradation because of the variable reflectivity during the scanning time will be higher if the scenario includes densely vegetated areas that are shaken by the wind. In this case, the main GB-SAR parameters (transmitted power, transmitted signal bandwidth, chirp rate, chirp period, scanning method, and time of observation) must be accurately chosen to minimize the cross-range blurring effect. It is obvious that it is mandatory to reduce the scanning time, but the transmitted energy has to be maintained for having the same SNR.

To assess the effect of the energy dispersion associated with the movement of the vegetation in the final image, a 

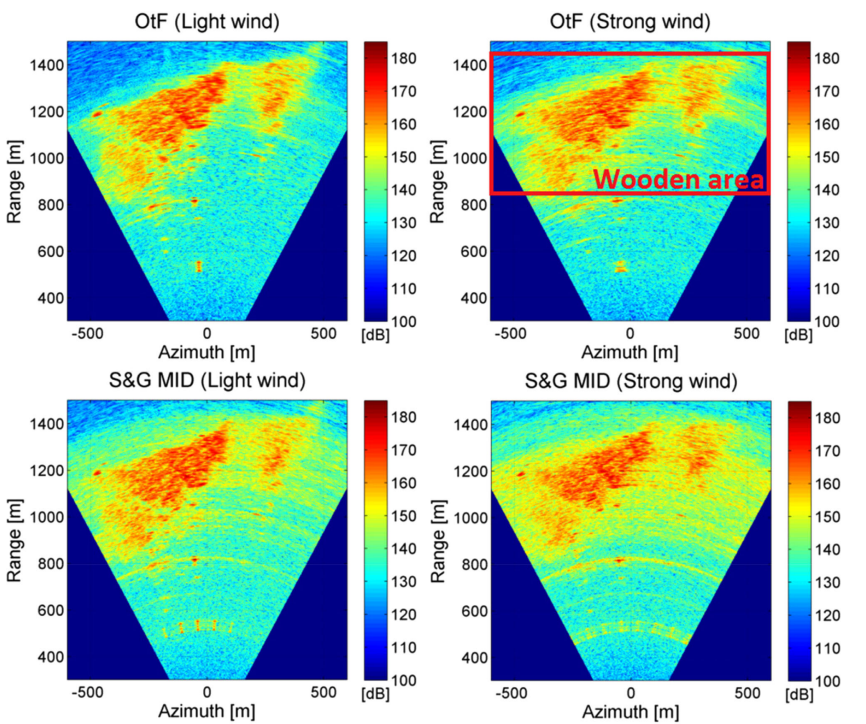

S\&G MID (Strong wind)

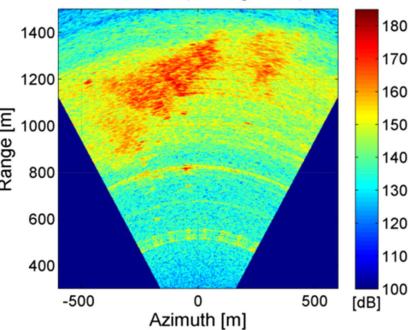

S\&G SLOW (Light wind)

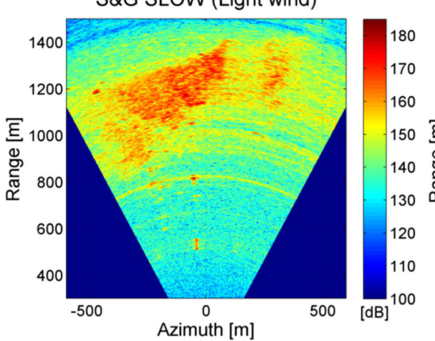

(a)

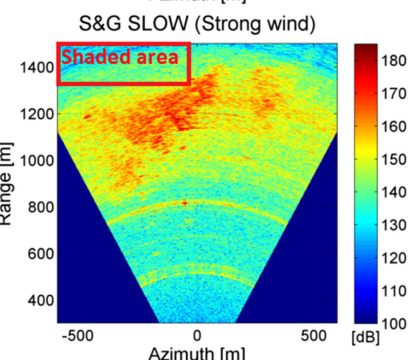

(b)

Fig. 9. SLC images in Cartesian coordinates for different operation modes and different wind blow speeds. (a) Light mean wind speed of $2 \mathrm{~m} / \mathrm{s}$. (b) Strong mean wind speed of $8.9 \mathrm{~m} / \mathrm{s}$.

compilation of SLC images is shown in Fig. 9. These images were obtained under different windblown conditions, with one OtF, and two S\&G scans, each with different scanning times. The mean wind speed for the measurements is $2 \mathrm{~m} / \mathrm{s}$ (light wind) and $8.9 \mathrm{~m} / \mathrm{s}$ (strong wind), corresponding to measurements 9 and 30 of the dataset. As introduced in Section II, there is a correlation between the scanning time and the blurring effect on the final image. The left column shows SLCs taken with light wind (low temperature and high humidity), while the right column is showing SLCs taken under strong wind (high temperature and low humidity). The best focused image corresponds to light wind and OtF scan, where the contours of different reflective surfaces can be clearly distinguished. On the other hand, a blurred image is obtained under strong wind blow and long aperture time. An indication that the cross-range blurring effect is affecting the quality of the images is the fact that in the SLCs corresponding to strong wind, the energy of the pixels associated with the back side of the mountain is increased when it should remain constant. As marked in the S\&G SLOW measurement in Fig. 9(b), the pixels of the left part of the image beyond $1200 \mathrm{~m}$ correspond to a shaded area. By comparing the measurements taken with the two S\&G modes, it can be seen that there are no significant differences between them. The relevance of the moment of measurement according to the type of scenario is noticeable. In this case, the scenario contains static high reflective (buildings and
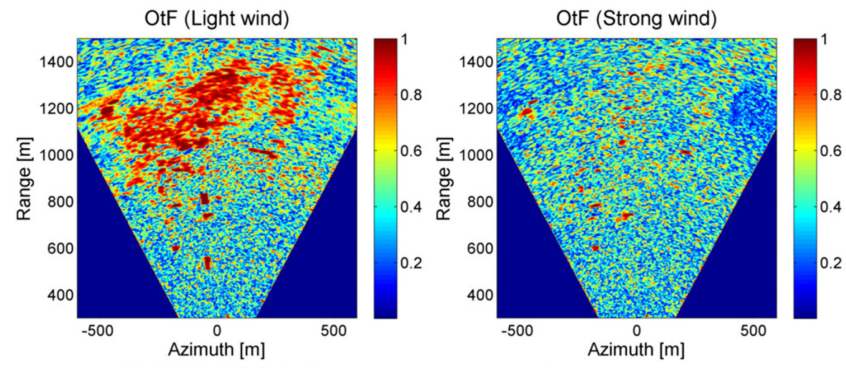

S\&G MID (Light wind)

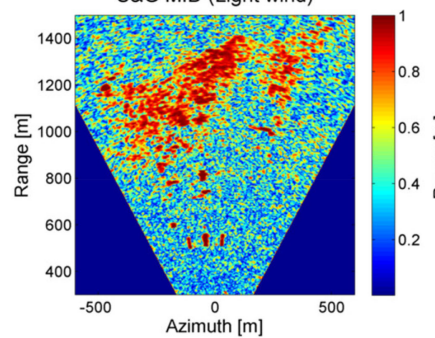

S\&G MID (Strong wind)

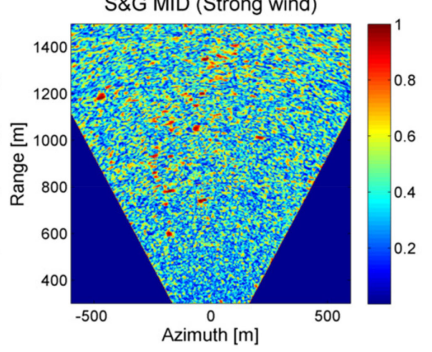

S\&G SLOW (Light wind)

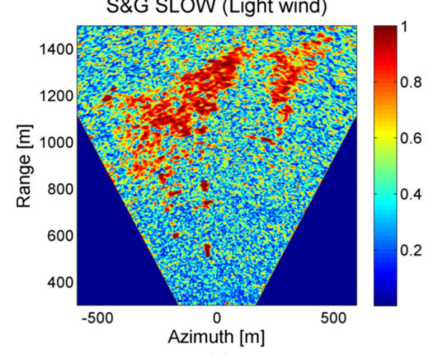

(a)

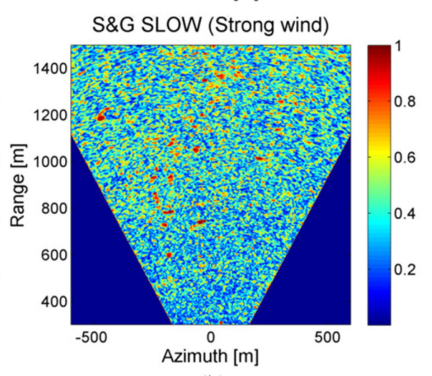

(b)
Fig. 10. Coherence $|\gamma|$ of differential interferometric images for different operation modes and different wind blow speeds. (a) Light mean wind speed of $2 \mathrm{~m} / \mathrm{s}$. (b) Strong mean wind speed of $8.9 \mathrm{~m} / \mathrm{s}$.

urban structures) and distributed scatterers (trees and bushes). Latter scatterers have a short-term variable reflectivity due to the wind during the scanning time, which is normal in scenarios used for GB-SAR applications. Short-term variable reflectivity together with long scanning time strongly increases the image cross-range blurring, as shown in the simulations in Fig. 2.

\section{Coherence Map Evaluation}

After analyzing the different SLC images, some temporal differential interferometric images based on the same master image have been generated. The aim was to evaluate how the coherence is affected by the fact of having a scenario with high coherent scatterers surrounded by vegetated areas with shortterm variable reflectivity.

Fig. 10 shows the coherence $|\gamma|$ of six differential interferometric images obtained with the OtF, S\&G MID, and the S\&G SLOW modes for two different mean wind speeds of 2 and $8.9 \mathrm{~m} / \mathrm{s}$. It can be observed that for light wind speed, the number of coherent pixels, the ones that maintain a constant reflectivity over the time, is reduced as the duration of the scanning time increases, overcoat between the OtF and the $S \& G$ mode. In the measurements with strong wind speed, the number of coherent pixels has been drastically reduced for the three operation modes. Table II shows a summary of the evolution of the number 
TABLE II

EVALUATION OF THE NUMBER OF COHERENT PIXELS

\begin{tabular}{l|cccccc}
\hline \hline \multicolumn{4}{c}{ LIGHT WIND } & \multicolumn{2}{c}{ STRONG WIND } \\
\hline & OtF & $\begin{array}{l}\text { S\&G } \\
\text { MID }\end{array}$ & $\begin{array}{l}\text { S\&G } \\
\text { SLOW }\end{array}$ & OtF & $\begin{array}{l}\text { S\&G } \\
\text { MID }\end{array}$ & $\begin{array}{l}\text { S\&G } \\
\text { SLOW }\end{array}$ \\
\hline $\begin{array}{l}\text { Pixels with coherence } \\
\text { amplitude>0.6 }\end{array}$ & 90342 & 73821 & 70112 & 37174 & 34758 & 34571 \\
$\begin{array}{l}\text { Reduction respect OtF } \\
{[\%]}\end{array}$ & - & 18.28 & 22.39 & - & 6.5 & 7 \\
\hline \hline
\end{tabular}

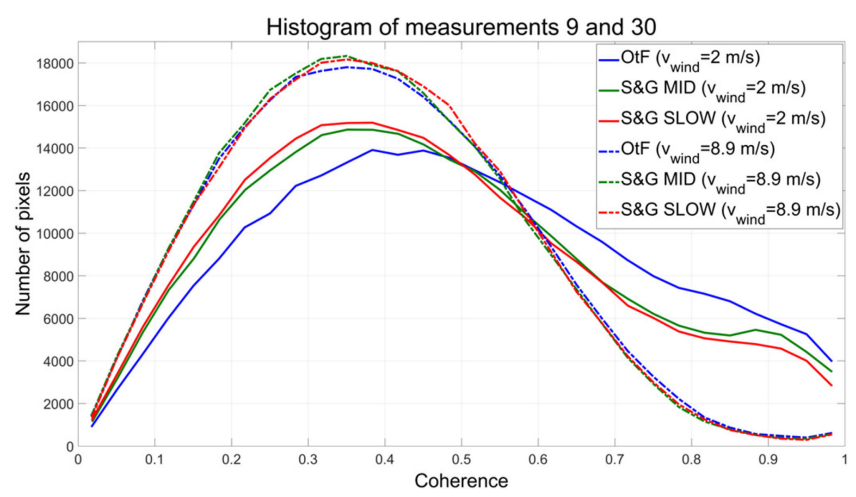

Fig. 11. Normalized histogram of the coherence $|\gamma|$ for two different mean wind speeds $v_{\text {wind }}=2$ and $8.9 \mathrm{~m} / \mathrm{s}$, and the three different operation modes.

of coherent pixels for the different operation modes and wind speeds.

Table II shows that, for the light wind measurements, the coherence map corresponding to the S\&G MID operation mode has a reduction of the $18.28 \%$ with respect to the OtF case in the density of coherent pixels with a coherence amplitude greater than 0.6. The S\&G SLOW mode has a reduction of $22.39 \%$ with respect to the OtF case. For the strong wind, the S\&G MID and the S\&G SLOW have a reduction of $6.5 \%$ and $7 \%$, respectively, with respect to the OtF case. Besides, the measurements under strong wind have a reduction in the number of coherent pixels with respect to the measurements under light wind of $58.8 \%$, $53.2 \%$, and $50.69 \%$ for the OtF, S\&G MID, and S\&G SLOW, respectively.

Fig. 11 shows the normalized histogram of the coherence $|\gamma|$ for two different mean wind speeds and the three different operation modes. It is clearly visible that for light wind conditions, the OtF scan method offers the highest number of pixels with an elevated coherence, while the two $S \& G$ scans are almost indistinguishable. On the other hand, with strong wind conditions, the three scanning modes have a similar performance with higher number of pixels with low coherence. Thus, after analyzing the results shown in Figs. 10 and 11, it has been concluded that for the scenarios containing or surrounded by vegetation and strong wind conditions, the reduction of the scanning time between the tree operation modes is not enough to avoid the blurring effect and the decorrelation between images.

After analyzing the differential interferometric images of the measurements realized during the two days of campaign, Fig. 12 exposes the evolution of the density of pixels in the image with a coherence value over a certain threshold, in this case, 0.6 . The plot clearly shows that the density of coherent pixels is higher for

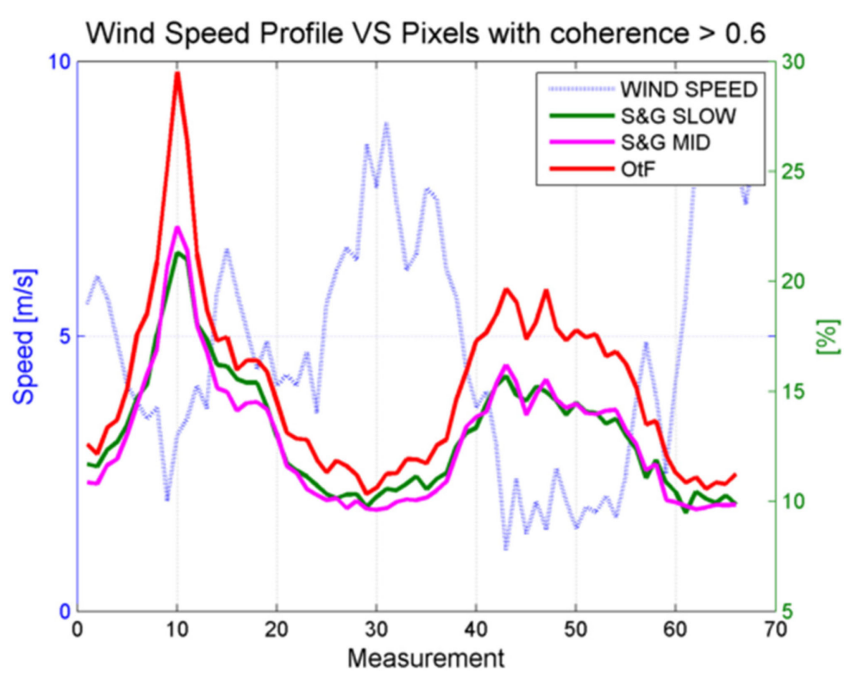

Fig. 12. Superposition of mean wind speed and the evolution of pixel density with coherence higher than 0.6 . The highest density is close to the master image. A strong correlation between coherent pixel density and mean wind speed is detected.

TABLE III

EVALUATION OF THE DOPPLER BANDWIDTH

\begin{tabular}{cccc}
\hline \hline $\begin{array}{c}\text { RESOLUTION } \\
{[\mathrm{M}]}\end{array}$ & \multicolumn{3}{c}{ DOPPLER BANDWIDTH (Hz) } \\
\hline & $\begin{array}{c}\text { GB-SAR } \\
(\mathrm{v}=0.5 \mathrm{~m} / \mathrm{s})\end{array}$ & $\begin{array}{c}\text { AIRBORNE } \\
(\mathrm{v}=100 \mathrm{~m} / \mathrm{s})\end{array}$ & $\begin{array}{c}\text { ORBITAL } \\
(\mathrm{v}=7500 \mathrm{~m} / \mathrm{s})\end{array}$ \\
\hline 15 & 0.03 & 6.6 & 500 \\
1.5 & 0.3 & 66 & 5000 \\
\hline \hline
\end{tabular}

short aperture times and that there is a strong correlation with the wind. As the test site is in part composed by vegetated areas in which the foliage moves with the effect of the wind, varying their reflectivity, the higher the wind speed during the acquisitions, the lower the number of density pixels with coherence over a certain threshold.

In GB-SAR systems, the effect of the energy spreading along the cross-range of adjacent cells must be taken into account because it can degrade the quality of the retrieved information. This can be seen, for instance, in the reduction of the amount of coherent pixels in the coherence maps. The spreading effect can be explained in terms of the equivalent Doppler bandwidth of the illuminated scene. For long scanning time, the particular geometry of the scenario forces a reduced Doppler bandwidth of a fraction of a cycle, while the equivalent frequency spreading of the variable scatterers is hundreds of $\mathrm{Hz}$. Because of that, these systems are affected by the blurring effect and the decorrelation between images can arise.

The blurring effect is not present in the same way in orbital SARs, but it could be noticeable in airborne SARs, depending on the Doppler bandwidth and the frequency spreading of the energy of the scatterers. In orbital SARs, the equivalent Doppler bandwidth is wider than the energy frequency spreading of deterministic and random moving scatterers. This is summarized in Table III that shows the evaluation of the Doppler bandwidth for a given range resolution as a function of the different SAR topologies. 


\section{CONCLUSION}

The potentials of GB-SAR sensors to monitor deformation episodes have been demonstrated during the last years. The high stability and flexibility of the sensor platform, in addition to their reduced cost compared to orbital platforms, make them ideal to detect changes in small-scale areas of observation by means of GB-SAR interferometry. Nevertheless, some limitations in the performance of GB-SAR systems must be pointed out. This paper has studied some of these limitations, directly related to the short aperture length together with the relatively long scanning time and the change in the reflectivity of the scenario during the measurement. These changes of reflectivity, due to moving vegetation, can be forced by atmospheric phenomena, concretely the wind. The main effects assessed in the present paper are image blurring, decorrelation, and hence, an important coherence degradation. In this context, it has been concluded that the retrieved information from scenarios surrounded by vegetation can be deteriorated, since pixels associated with moving scatterers alter the reflectivity of neighboring pixels in the same radial range. Taking into account that GB-SAR systems are mainly used to detect and monitor changes in heterogeneous scenarios by means of the coherence maps, the degradation of the quality of these maps is an important drawback that has to be minimized. By means of working with the OtF and S\&G operation modes, it has been confirmed that to reduce the blurring effect in the cross-range direction, it is necessary to reduce the scanning time while maintaining the total transmitted energy. Thus, a readjustment of the chirp repetition pulse is mandatory to ensure that the spatial Nyquist criterion is accomplished. Nevertheless, despite an important reduction of scanning time to seconds, under strong wind conditions no improvement is reached, so it is clearly preferable to take measurements under stable atmosphere.

A mathematical description of the GB-SAR cross-range focusing process has been developed, which permitted the analysis and characterization of the effect in the image reconstruction process of having moving vegetation surrounding the area under study. Periodic changes in scatterer reflectivity imply the apparition of cross-range replicas of the scatterer, while the random ones force image blurring and image decorrelation. A new term in the differential interferometric coherence $\gamma_{\text {blur }}$ that takes into account the image blurring has been introduced. This effect can be considered as noise, having a direct dependence with $\mathrm{SIR}_{e}$. It is beyond the scope of this work to characterize the interference signal $\mathrm{SIR}_{e}$ as a function of the moving scatterers. In this context, some general explanations of different particular cases have been pointed out.

The RISKSAR-X GB-SAR sensor has been used to experimentally validate the effects of the wind on the retrieved data while measuring scenarios containing urban and vegetated areas. The experimental results have shown a decorrelation at $\mathrm{X}$-band between data acquired at different instants, which implies a decrease of the coherence of the overall image.

Despite technical complexity, new GB-SAR systems based on MIMO represent a promising solution to overcome the windinduced scatterer motion effect on SAR imaging, since they drastically reduce the scan time, being able to synthesize the aperture without mechanical movement of the antennas. Nevertheless, both GB-SAR approaches must be considered as complementary as they may address different applications or needs of final users.

\section{REFERENCES}

[1] D. Massonnet and K. L. Feigl, "Radar interferometry and its application to changes in the Earth's surface," Rev. Geophys., vol. 36, no. 4, pp. 441-500, 1998.

[2] R. Bürgmann et al., "Synthetic aperture radar interferometry to measure Earth's surface topography and its deformation," Anпи. Rev. Earth Planet. Sci., vol. 28, no. 1, pp. 169-209, 2000.

[3] D. Leva, G. Nico, D. Tarchi, J. Fortuny-Guasch, and A. J. Sieber, "Temporal analysis of a landslide by means of a ground-based SAR interferometer," IEEE Trans. Geosci. Remote Sens., vol. 41, no. 4, pp. 745-752, Apr. 2003.

[4] L. Pipia, X. Fabregas, A. Aguasca, and C. Lopez-Martinez, "Polarimetric temporal analysis of urban environments with a ground-based SAR," IEEE Trans. Geosci. Remote Sens., vol. 51, no. 4, pp. 2343-2360, Apr. 2013.

[5] A. Aguasca, A. Broquetas, J. J. Mallorqui, and X. Fabregas, “A solid state L to X-band flexible ground- based SAR system for continuous monitoring applications," in Proc. IEEE Int. Geosci. Remote Sens. Symp., Sep. 2004, pp. 757-760.

[6] L. Pipia, "Polarimetric differential SAR interferometry with ground-based sensors," Ph.D. dissertation, , Univ. Politècnica de Catalunya, Barcelona, Spain, 2011.

[7] R. Iglesias et al., "Ground-based polarimetric SAR interferometry for the monitoring of terrain displacement phenomena-Part I: Theoretical description," IEEE J. Sel. Top. Appl. Earth Obs. Remote Sens., vol. 8, no. 3, pp. 980-993, Mar. 2015.

[8] J. Klare, O. Saalmann, H. Wilden, and A. R. Brenner, "First experimental results with the imaging MIMO radar MIRA-CLE X," in Proc. 8th Eur. Conf. Synth. Aperture Radar, Aachen, Germany, 2010, pp. 1-4.

[9] J. Klare and O. Saalmann, "MIRA-CLE X: A new imaging MIMO-radar for multi-purpose applications," in Proc. 7th Eur. Radar Conf., Paris, France, 2010, pp. 129-132.

[10] J. K. Biallawons, R. Klenke, and R. Panhuber, "MIMO concept for the imaging radar of the radar warning and information system RAWIS," in Proc. 11th Eur. Conf. Synth. Aperture Radar, Hamburg, Germany, 2016, pp. 1-4.

[11] K. Morrison and M. L. Williams, "High resolution PolInSAR with the ground-based SAR (GB-SAR) system: Measurement and modelling," in Proc. Int. Geosci. Remote Sens. Symp., Seoul, South Korea, Jul. 2005, vol. 2, pp. 1105-1108.

[12] K. Morrison, "Towards a quantitative understanding of the effects of wind motion on airborne and satellite SAR imagery of vegetation," in Proc. Int. Geosci. Remote Sens. Symp., Toulouse, France, Jul. 2003, pp. 3281-3283.

[13] K. Morrison and L. Oldfield, "The effect of target motion on ISAR imagery," in Proc. Antenna Meas. Techn. Assoc., Cleveland, OH, USA, Nov. 2002, pp. 307-312.

[14] P. Lombardo and J. B. Billingsley, "A new model for the Doppler spectrum of windblown radar ground clutter," in Proc. IEEE Radar Conf. Radar Next Millennium (Cat. no. 99CH36249), 1999, pp. 142-147.

[15] D. Leva, G. Nico, D. Tarchi, J. Fortuny-Guasch, and A. J. Sieber, "Temporal analysis of a landslide by means of a ground-based SAR interferometer," IEEE Trans. Geosci. Remote Sens., vol. 41, no. 4, pp. 745-752, Apr. 2003.

[16] M. Pieraccini, L. Noferini, D. Mecatti, G. Macaluso, G. Luzi, and C. Atzeni, "Digital elevation models by a GBSAR interferometer for monitoring glaciers: The case study of Belvedere Glacier," in Proc. IEEE Int Geosci. Remote Sens. Symp., 2008, vol. 4, pp. IV-1061-IV-1064.

[17] L. Noferini et al., "Analysis of ground-based SAR data with diverse temporal baselines," IEEE Trans. Geosci. Remote Sens., vol. 46, no. 6, pp. 1614-1623, Jun. 2008.

[18] L. Noferini et al., "Using GB-SAR technique to monitor slow moving landslide," Eng. Geol., vol. 95, no. 3, pp. 88-98, 2007.

[19] D. Tarchi et al., "Landslide monitoring by using ground-based SAR interferometry: An example of application to the Tessina landslide in Italy," Eng. Geol., vol. 68, no. 1, pp. 15-30, 2003. 
[20] R. Iglesias et al., "Ground-based polarimetric SAR interferometry for the monitoring of terrain displacement phenomena-Part II: Applications," IEEE J. Sel. Top. Appl. Earth Obs. Remote Sens., vol. 8, no. 3, pp. 994 1007, Mar. 2015.

[21] R. Iglesias et al., "Atmospheric phase screen compensation in groundbased SAR with a multiple-regression model over mountainous regions," IEEE Trans. Geosci. Remote Sens., vol. 52, no. 5, pp. 2436-2449, May 2014.

[22] S. Rödelsperger, A. Coccia, D. Vicente, and A. Meta, "Introduction to the new metasensing ground-based SAR: Technical description and data analysis," in Proc. IEEE Int. Geosci. Remote Sens. Symp., 2012, pp. 4790-4792.

[23] M. Soumekh, Synthetic Aperture Radar Signal Processing With MATLAB Algorithms. New York, NY, USA: Wiley, 1999.

[24] R. J. C. Middleton, "Dechirp-on-receive linearly frequency modulated radar as a matched-filter detector," IEEE Trans. Aerosp. Electron. Syst., vol. 48, no. 3, pp. 2716-2718, Jul. 2012.

[25] B. C. Barber, "Some effects of target vibration on SAR images," in Proc. 7th Eur. Conf. Synth. Aperture Radar, 2008, pp. 1-4.

[26] F. Rocca, "Modeling interferogram stacks," IEEE Trans. Geosci. Remote Sens., vol. 45, no. 10, pp. 3289-3299, Oct. 2007.

[27] R. Raney, "Wave orbital velocity, fade, and SAR response to azimuth waves," IEEE J. Ocean. Eng., vol. OE-6, no. 4, pp. 140-146, Oct. 1981.

[28] R. Touzi, A. Lopes, J. Bruniquel, and P. W. Vachon, "Coherence estimation for SAR imagery," IEEE Trans. Geosci. Remote Sens., vol. 37, no. 1, pp. 135-149, Jan. 1999.

[29] H. A. Zebker and J. Villasenor, "Decorrelation in interferometric radar echoes," IEEE Trans. Geosci. Remote Sens., vol. 30, no. 5, pp. 950-959, Sep. 1992.

[30] L. Pipia, X. Fabregas, A. Aguasca, and C. Lopez-Martinez, "Atmospheric artifact compensation in ground-based DInSAR applications," IEEE Geosci. Remote Sens. Lett., vol. 5, no. 1, pp. 88-92, Jan. 2008.

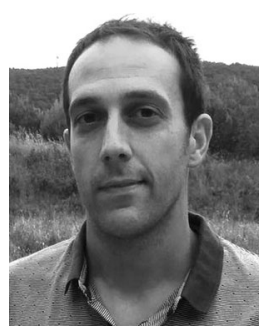

Marc Lort (M'xx) was born in Sabadell, Spain, in 1986. He received the B.Sc. degree in telecommunication engineering from the Universitat Autònoma de Barcelona, Barcelona, Spain, in 2010, and the M.Sc. degree from the Signal Theory and Communications Department, Universitat Politècnica de Catalunya, Barcelona, Spain, in 2014. He is currently working toward the Ph.D. degree, focused on multidimensional synthetic aperture radar (SAR) for the Earth observation.

His main research interests include the development and test of SAR systems integrated into unmanned aerial vehicles.

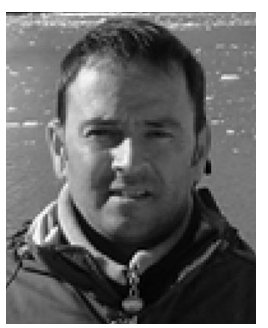

Albert Aguasca (S'90-M'94) was born in Barcelona, Spain, in 1964. He received the M.Sc. and $\mathrm{Ph} . \mathrm{D}$. degrees in telecommunication engineering from the Universitat Politècnica de Catalunya (UPC), Barcelona, Spain, in 1989 and 1993, respectively.

Since 1995, he has been an Associate Professor with the School of Telecommunications Engineering, UPC. His teaching activities involve RF and microwave circuits for communications and radio navigation systems. He has authored or co-authored more than 40 papers on microwave synthetic aperture radar (SAR), radiometer systems, and microwave circuits. His research interests include the design and development of SAR and microwave radiometer systems for unmanned aerial vehicle platforms.

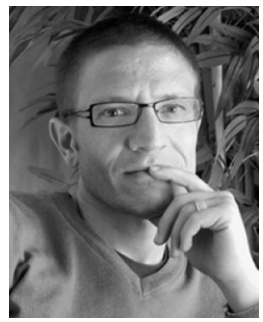

Carlos López-Martínez (S'97-M'04-SM'11) received the M.Sc. degree in electrical engineering and the Ph.D. degree from the Universitat Politècnica de Catalunya, Barcelona, Spain, in 1999 and 2003, respectively.

From October 2000 to March 2002, he was with the Frequency and Radar Systems Department, HR, German Aerospace Center, Oberpfaffenhofen, Germany. From June 2003 to December 2005, he was with the Image and Remote Sensing Group SAPHIR Team, Institute of Electronics and Telecommunications of Rennes (CNRS UMR 6164), Rennes, France. In January 2006, he as a Ramón-y-Cajal Researcher joined the Universitat Politècnica de Catalunya, Barcelona, Spain, where he is currently an Associate Professor in the area of remote sensing and microwave technology. He has authored or co-authored more than 100 articles in journals, books, and conference proceedings in the radar remote sensing and image analysis literature. His research interests include synthetic aperture radar (SAR) and multidimensional SAR, radar polarimetry, physical parameter inversion, digital signal processing, estimation theory, and harmonic analysis.

Dr. López-Martínez is an Associate Editor for the IEEE Journal OF SELECTED TOPICS IN APPLIED EARTH OBSERVATIONS AND REMOTE SENSING and he served as a Guest Editor for the EURASIP Journal on Advances in Signal Processing. He has organized different invited sessions in international conferences on radar and SAR polarimetry. He has presented advanced courses and seminars on radar polarimetry to a wide range of organizations and events. $\mathrm{He}$ was a recipient of the Student Prize Paper Award at the EUSAR 2002 Conference and co-authored the paper awarded with the First Place Student Paper Award at the EUSAR 2012 Conference. He has also received the IEEE-GRSS 2013 GOLD Early Career Award.

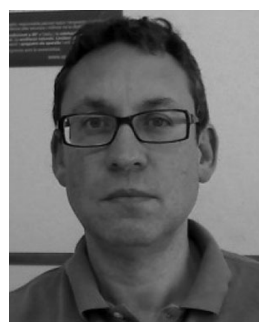

Xavier Fabregas (M'xx) received the B.S. degree in physics from Barcelona University, Barcelona, Spain, in 1988, and the Ph.D. degree in applied sciences from the Universitat Politecnica de Catalunya (UPC), Barcelona, Spain, in 1995.

In 1990, he joined the Photonic and Electromagnetic Engineering Group, Signal Theory and Communications Department, UPC. Since 1996, he has been an Associate Professor with UPC. In 2001, he spent an eight-month sabbatical with the HR Institute of the German Aerospace Agency, Oberpfaffenhofen. He has authored or co-authored 32 refereed papers in international journals, 135 conference proceedings, and has received a patent. His current research interests include radar polarimetry, polarimetric-retrieval algorithms, polarimetric calibration, polarimetric speckle models, ground-based synthetic aperture radar (SAR) sensors and their applications, and time series for multidimensional SAR data applications for urban and terrain deformation monitoring.

Dr. Fabregas is a Reviewer in several international journals. 